\title{
Synergistic Sorafenib with Jianpi Huayu Decoction Resulted in Tumor Regression and Prevented Adverse Events in Hepatocellular Carcinoma by Remodeling the Gut Microbiota
}

\section{Xue Deng}

Southern Medical University

\section{Yingjie Xie}

General Hospital of Guangzhou Military Command of PLA: People's Liberation Army General Hospital of Southern Theatre Command

Yantong Lu

Guangzhou University of Chinese Medicine

\section{Xueyan Li}

Southern Medical University

\section{Xuan Guo}

South China University of Technology

\section{Yuan Zhang}

Southern Medical University

Juze Lin

Guangdong General Hospital: Guangdong Provincial People's Hospital

Wei Tan

Guangdong Provincial People's Hospital

Changjun Wang ( $\nabla$ gzwchj@126.com )

Guangdong Provincial People's Hospital https://orcid.org/0000-0003-0420-4679

\section{Xuhui Huang}

Guangdong Provincial People's Hospital

\section{Research}

Keywords: Hepatocellular carcinoma, Jianpi Huayu, Decoction, Sorafenib, Microbiota.

Posted Date: September 2nd, 2021

DOI: https://doi.org/10.21203/rs.3.rs-844760/v1 
License: (c) (i) This work is licensed under a Creative Commons Attribution 4.0 International License. Read Full License 


\section{Abstract}

Background: There is an urgent need for effective treatments for hepatocellular carcinoma (HCC). Sorafenib is first-line treatment for HCC, which has a modest efficacy due to severe adverse effects(AE), acquired resistance and others. Combination therapy may be able to overcome this limitation. Jianpi Huayu Decoction (JHD) is a traditional Chinese medicine formulation, which has been shown to be effective as an alternative and complementary therapy of HCC. We investigated the synergistic effect of JHD with sorafenib by a xenograft model.

Methods: Growth of mouse-derived HCC cells and adverse events(AE) of treatment were evaluated in a subcutaneous model with JHD and clinical-dose sorafenib combination treatment. Diarrhea, the most frequently reported $\mathrm{AE}$, was evaluated by diarrhea score. The gut microbiota(GM) composition of the mice was analyzed by Illumina NovaSeq.

Results: JHD administration in mice synergistically enhanced the anti-tumor response, thereby suppressing HCC, and prevented occurrence of the most common AEs(diarrhea and body weight loss) of sorafenib. Sorafenib induced increased proinflammatory GM(Helicobacter) which promoted the progression of HCC and against anti-tumor treatment. JHD reduced the abundance of anti-inflammatory microbiota Muribaculum, Fusicatenibacter, and Dorea. Following the modulation of GM, the proinflammatory signaling interleukin 6/signal transducer and activator of transcription-3 pathway was downregulated by JHD.

Conclusions: Our finding suggested that differences in the microbial gut flora may modulate resistance to sorafenib through IL-6/STAT3 signaling. JHD with microbiota modulation properties could potentiate sorafenib and provided a promising approach for HCC treatment.

\section{Introduction}

Hepatocellular carcinoma (HCC) is the third leading cause of cancer-related mortality[1]. Curative therapies, such as resection, ablation, or transplantation, are only available to individuals who are diagnosed at an early stage. There are presently no effective treatment choices for people with advanced $\mathrm{HCC}[2]$. Sorafenib is the first FDA-approved first-line medication for advanced HCC, although it offers very moderate survival improvements[3]. Cancer immunotherapy is promising for HCC, but it should not be used alone. Immunotherapy has not outperformed sorafenib in terms of efficacy[4]. Due to the lack of more efficient approaches, the 5-year survival rate of HCC is only $18 \%[5]$, further emphasizing the urgent need to employ novel intervention strategies.

Jianpi Huayu Decoction (JHD) is a traditional Chinese medicine(TCM) formulation. It is used as an alternative and complementary therapy of liver-cancer. Previously, we showed that JHD inhibited the progression of $\mathrm{HCC}$ by inducing apoptosis[6] and immunomodulation[7]. Meanwhile, we noticed that the tumor-bearing mice treated with JHD were stronger than the mice with vehicle. Sorafenib is widely used for advanced HCC therapy in clinical, although $30 \%$ of patients can benefit from it due to severe adverse 
effects $(\mathrm{AE})$, acquired resistance, immunosuppressive microenvironment and others[8]. Diarrhea is the most common $A E(55 \%)$, as well as the main cause of $A E$ related dose reduction/interruption $(3 \%-5 \%)$ of sorafenib, which leads to body weight loss and therapy tolerance[9, 10]. The management of diarrhea caused by sorafenib is insufficient and a novel approach is needed. Combined treatment presents auspicious therapeutic strategies and is widely investigated in preclinical and clinical research. In term of effectiveness of JHD on improvement of body weight and inhibition of tumor, we speculate that sorafenib combined with JHD is a potential strategy. Therefore, we investigate whether JHD enhances the efficacy of sorafenib and how it works.

\section{Materials And Methods \\ 2.1. Cell line and cell culture}

Cell line BNL 1ME A.7R.1 (referred as BNL in this study) from BALB/C mouse, sensitive to sorafenib, was obtained from Shanghai Xin Yu Biotechnology(Shanghai, China), and maintained in Dulbecco's minimal essential medium (DMEM) (Gibco) supplemented with $10 \%$ heat-inactivated $\left(56^{\circ} \mathrm{C}, 30 \mathrm{~min}\right)$ fetal bovine serum (Gibco) and $100 \mathrm{U} / \mathrm{mL}$ penicillin, $100 \mu \mathrm{g} / \mathrm{mL}$ streptomycin (Gibco), L-glutamin (2 mM) in a humidified atmosphere of $5 \% \mathrm{CO}_{2}$ at $37^{\circ} \mathrm{C}$.

\subsection{JHD preparation}

$\mathrm{JHD}$ is a combination of six medicinal herbs: Atractylodes macrocephala, Curcuma zedoaria, Radix Sophorae flavescentis, Citrus medica, Hedyotis diffusa, Radix Sophorae flavescentis at a rate of 3:3:3:5:5:5 (w/w/w/w/w/w). The six herbs of JHD were purchased from Shou Yi Zhen Yuan (Xiamen, China) and were identified by two experienced pharmacists. For extraction of JHD, the herbs were first soaked in deionized water at tenfold volume $(\mathrm{v} / \mathrm{w}) 30 \mathrm{~min}$ and then extracted by decoction two times, $1 \mathrm{~h}$ for the first time and $45 \mathrm{~min}$ for the second time with sixfold volume of deionized water to herbs $(\mathrm{v} / \mathrm{w})$. After filtration, the solution was evaporated under reduced pressure to obtain an extract, and then the extract was desiccated to powder at $60^{\circ} \mathrm{C}$ and stored at $-80^{\circ} \mathrm{C}$ for use.

\subsection{Animals and treatment}

Male BALB/c mice ( 5 weeks) were purchased from the Animal Center of Southern Medical University. They were maintained under specific pathogen-free conditions, at $22-26^{\circ} \mathrm{C}$, relative humidity of $55 \pm 5 \%$, and a $12 \mathrm{~h}$ light-dark cycle. A subcutaneous tumor-bearing model was established by infecting $5 \times$ $10^{6} / 100 \mu \mathrm{L}$ of BNL cells in the right flank. When the tumor reached a diameter of $100-200 \mathrm{~mm}^{3}$, mice were divided randomly into four group JHD, sorafenib, JHD + sorafenib and vehicle with five mice in each group. Sorafenib (Nexavar ${ }^{\mathrm{TM}}$ ) was purchased from MedChemExpress (Monmouth Junction, NJ,USA) and dissolved in vehicle ( $10 \%$ dimethyl sulfoxide $+40 \%$ polyethylene glycol $300+5 \%$ Tween $-80+45 \%$ saline). JHD was prepared as "2.2" described. Mice were fed with vehicle $(10 \mathrm{~mL} / \mathrm{kg})$ and JHD $(24.96 \mathrm{~g} / \mathrm{kg})$ or sorafenib (30 mg/kg/day) or a combination of JHD and sorafenib, or vehicle $(100 \mu \mathrm{L} / 10 \mathrm{~g})$ by gavage. 
The study protocol was approved by the Animal Care and Use Committee of Southern Medical University (Guangzhou, China).

\subsection{Gut microbiota analysis}

Mice fecal samples were collected ( 23 days after implantation)and stored at $-80^{\circ} \mathrm{C}$ immediately. Total genome DNA from samples was extracted using CTAB-SDS method. 16S rRNA genes were amplified used the specific primer with the barcode. PCR products was purified with GeneJET Gel Extraction Kit(Thermo Scientific). Sequencing libraries were established using Illumina TruSeq DNA PCR-Free Library Preparation Kit (Illumina, USA) following manufacturer's instructions and index codes were added. The library quality was assessed on the Qubit@ 2.0 Fluorometer (Thermo Scientific) and Agilent Bioanalyzer 2100 system. At last, the library was sequenced on an Illumina NovaSeq platform and 250 bp paired-end reads were generated.

Paired-end reads from the original DNA fragments were merged by using FLASH[11], and was assigned to each sample according to the unique barcodes. Sequences were analyzed using QIIME[12] software package (Quantitative Insights Into Microbial Ecology), and in-house Perl scripts were used to analyze alpha and beta diversity. First, reads were filtered by QIIME quality filters. Then we used pick_de_novo_otus.py to pick operational taxonomic units (OTUs) by making OTU table. Sequences with $\geq 97 \%$ similarity were assigned to the same OTUs. We picked representative sequences for each OTU and used the RDP classifier[13] to annotate taxonomic information for each representative sequence. We rarified the OTU table and calculated three metrics Chao1 and Shannon index. We used weighted unifrac, calculated by QIIME, for principal coordinate analysis (PCOA). Significance test was conducted with some statistical analysis methods, including T-test, similarity percentages breakdown (SIMPER) and linear discriminant analysis effect size(LEfSe).

\subsection{Western-Blot analysis and qRT-PCR}

Total protein and RNA was extracted using extraction kit following the manufacturer's instructions. Protein was quantified, separated, transferred and blocked as previous description. The membranes were incubated with primary antibodies ( $\beta$-actin, affinity $1: 5000$; STAT3, CST $1: 1000$; pSTAT3, CST $1: 2000$; iNOS, CST 1 : 2000). Protein bands were quantified using ImageJ software with $\beta$-actin as the internal control. The expression of mRNA was measured via qRT-PCR using a SYBR PrimeScript RT-PCR Kit (Takara Bio, Shiga, Japan) in accordance with the manufacturer's instructions. We used $\beta$-actin as an internal control. The primers used are listed in Table 1. We calculated relative mRNA levels based on the $C t$ values and normalized using $\beta$-actin expression. 
Table 1

Sequence of Primers Used in Real-Time Experiments (related to Experimental Procedures)

\begin{tabular}{|lll|}
\hline Gene & Forward Primer & Reverse Primer \\
\hline IL-6 & TAGTCCTTCCTACCCCAATTTCC & TTGGTCCTTAGCCACTCCTTC \\
\hline$\beta$-actin & GGCTGTATTCCCCTCCATCG & CCAGTTGGTAACAATGCCATGT \\
\hline
\end{tabular}

2.6.Hematoxylin-eosin staining(H\&E), immunohistochemistry(IHC) and immunofluorescence(IF)

For $\mathrm{H} \square \mathrm{E}$, the slices were dipped into hematoxylin reagent (Sangon Biotech) for $10 \mathrm{~min}$, rinsed with distilled water for $10 \mathrm{~min}$, and dehydrated with anhydrous ethanol for $5 \mathrm{~min}$. Finally, the slices were dipped into an eosin dye solution (Sangon Biotech) for 3 min, dehydrated with anhydrous ethanol for $3 \mathrm{~min}$, and sealed with neutral gum. All slices were observed under an inverted microscope and images were collected. For immunohistochemistry, the tumor tissue slices were incubated with PCNA (1:500, abcam ab92552), CD31 (1:2000, abcam ab182981), VEGFA (1:250, abcam ab52917) antibody and with Horseradish peroxidaseconjugated secondary antibody. For immunofluorescence, the tumor tissue slices were incubated with IL6 primary antibody (1:200, CST 12912), JAK2(1:250, abcam ab108596) and with Alexa Fluor 488/594conjugated secondary antibody (Abbkine, Wuhan, CA).

\subsection{High-performance liquid chromatography coupled with mass spectrometry (HPLC-MS)}

JHD water extract was analyzed by high-performance liquid chromatography with hybrid linear ion trap Orbitrap mass spectrometry (HPLC-LTQ/Orbitrap) equipped with an ACQUITY BEH C18 column (100 mm $\times 2.1 \mathrm{~mm}$ i.d., $1.7 \mu \mathrm{m}$; Waters, Milford, USA). The mobile phases consisted of $0.1 \%$ formic acid in water (solvent $\mathrm{A}$ ) and $0.1 \%$ formic acid in acetonitrile: isopropanol $(1: 1, \mathrm{v} / \mathrm{v})$ (solvent $\mathrm{B})$. The sample injection volume was $2 \mathrm{uL}$ and the flow rate was set to $0.4 \mathrm{~mL} / \mathrm{min}$. The mass spectrometric data was collected using a UHPLC-Q Exactive Mass Spectrometer (Thermo, USA) equipped with an electrospray ionization source operating in either positive or negative ion mode. The optimal conditions were set as followed: Aus gas heater temperature, $400^{\circ} \mathrm{C}$; Sheath gas flow rate $40 \mathrm{psi}$; Aus gas flow rate 30 psi; ion-spray voltage floating, $-2800 \mathrm{~V}$ in negative mode and $3500 \mathrm{~V}$ in positive mode, respectively; Normalized collision energy, 20-40-60V rolling for MS/MS. Data acquisition was performed with the Data Dependent Acquisition mode. The detection was carried out over a mass range of 70-1050 m/z.

\subsection{Statistical analysis}

Tumor weight, body weight, the expression of mRNA and protein, the relative abundance of gut microbiota and the numbers of MDSCs were analyzed using graphpad prsim 5 (GraphPad Software, Inc. USA). Part of the $16 \mathrm{~S}$ rRNA analysis and spearman correlation was carried out in R software. All data was expressed as mean \pm S.E.M. ANOVA and $t$-test were used when data accorded with normal distribution and homogeneity of variance. Tukey test was used multiple comparisons. A p-value $<0.05$ indicated that the difference was statistically significant. 
Results

\subsection{Identification of components of JHD}

The components of JHD were identified by HPLC-MS. Ten potential compounds, that is, Citropten 7, Formononetin, 1-Kestose, Biochanin A, Xanthohumol, Cytisine, Ferulic acid, Gallic acid Hesperetin, and Quercetin 3-0-glucoside were identified (Supplement 1A, Table 2), which were lined to what we identified before[7]. The characterizations and sources of these compounds are listed in Table 2.

Table 2

Identification of potential components of JHD.

\begin{tabular}{|lllll|}
\hline & Identification & Molecular weight M/Z & Molecular Formula & Retention time \\
\hline 1 & Citropten & 207.0654332 & $\mathrm{C} 11 \mathrm{H} 1004$ & 5.915317 \\
\hline 2 & Formononetin & 269.0811284 & $\mathrm{C} 16 \mathrm{H} 12 \mathrm{O} 4$ & 4.686867 \\
\hline 3 & 1 -Kestose & 543.132835 & $\mathrm{C} 18 \mathrm{H} 32 \mathrm{O} 16$ & 0.616733 \\
\hline 4 & Biochanin A & 283.0617134 & $\mathrm{C} 16 \mathrm{H} 12 \mathrm{O} 5$ & 3.5596 \\
\hline 5 & Xanthohumol & 355.1545057 & $\mathrm{C} 21 \mathrm{H} 22 \mathrm{O} 5$ & 6.086867 \\
\hline 6 & Cytisine & 191.1180483 & $\mathrm{C} 11 \mathrm{H} 14 \mathrm{~N} 20$ & 0.67545 \\
\hline 7 & Ferulic acid & 193.0503398 & $\mathrm{C} 10 \mathrm{H} 1004$ & 4.934583 \\
\hline 8 & Gallic acid & 169.0137157 & $\mathrm{C} 7 \mathrm{H} 605$ & 1.447133 \\
\hline 9 & Hesperetin & 301.0723318 & $\mathrm{C} 16 \mathrm{H} 1406$ & 4.408767 \\
\hline 10 & Quercetin 3-O-glucoside & 465.1035356 & $\mathrm{C} 21 \mathrm{H} 20012$ & 3.540883 \\
\hline
\end{tabular}

\subsection{JHD inhibited the growth of tumor and enhanced the therapeutic effect of sorafenib in vivo.}

To investigate the combined effect of JHD and sorafenib, we determined the anti-HCC effect of sorafenib at a clinical dose $(30 \mathrm{mg} / \mathrm{kg})$ in syngeneic mouse models. Obviously, sorafenib inhibited tumor growth, and the combination treatment of sorafenib and JHD showed more dramatically suppression than JHD alone or sorafenib alone(Fig. 1A, B). We also weighed the tumor tissue excised from tumor-bearing mice, and the weight of tumor from the JHD group was lower than that of the vehicle group (Fig. 1C). To further confirmed the synergistic effect of JHD on sorafenib, we assessed the proliferation and angiogenesis of tumor tissue by staining with proliferating cell nuclear antigen (PCNA), CD31 and vascular endothelial growth (VEGF) stain. JHD had slight effect on the proliferation of tumor cells, whereas the sorafenib combined with JHD strongly suppressed cell proliferation in vivo(Fig. 1D). Sorafenib significantly inhibited the expression of VEGFA(Fig. 1G), which is one of the target of sorafenib[14], and decreased tumor angiogenesis(as indicated by reduced microvessel density in tumors). The combined of JHD and 
sorafenib further inhibited tumor angiogenesis (Fig. 1F). These results indicated that JHD induced a synergistic antitumor effect when combined with sorafenib for HCC treatment.

\subsection{JHD protected occurrences of sorafenib-induced diarrhea and subsequent occurrences of body weight loss.}

Although tumor growth was efficiently suppressed, sorafenib led to diarrhea and body weight loss, suggesting that side effects were induced. In clinical, $\sim 80 \%$ of patients treated with sorafenib suffer AEs, such as diarrhea, body weight loss, hand-foot skin reaction, and hypophosphatemia[3, 9].

The most frequent AEs (any grade) were diarrhea. Body weight loss is common in patients experienced diarrhea. In this study, significant reduction in body weight of mice treated with sorafenib was observed. Treatment of JHD exhibited remarkably improvement on the loss of body weight and diarrhea control induced by sorafenib. Moreover, we observed a decrease of diarrhea accompanied by less body weight loss in the mice treated with the combination of sorafenib and JHD compared with that of treatment of sorafenib alone(Fig. 2A, B).

\subsection{Sorafenib induced increased proinflammatory microbiota}

Emerging evidences suggest that GM plays a vital role in progression of $\mathrm{HCC}[15]$ and cancer immunotherapy[16]. Meanwhile, microbiota dysbiosis, as indicated by drastic bacterial population changes at the phylum and genus levels is associated with a higher risk of diarrhea and can be a consequence of diarrhea. Then we try to understand these findings by the changes of GM. Overall, 82,887 useable reads and 1,487 operational taxonomic units (OTUs) were obtained from 20 samples(Supplement 2). The relative abundance of GM was analyzed at the phylum level, Bacteroidota and Firmicutes accounted for $₫ 90 \%$ of the total community of GM (Fig. 3A). We noted that Firmicutes/Bacteroidetes (F/B) ratio, associated with disease or imbalance in metabolism, [17, 18], was increased after treated with sorafenib, whereas had a reduction in the combination of sorafenib and JHD group(Fig. 3B).Then, we sought to determine if differences existed in the alpha diversity and beta diversity. The alpha diversity was significantly lower in the sorafenib group than that in the other three groups based on the Shannon index (Fig. 3C), and the beta analysis showed a opposite result with weighted unifrac(Fig. 3D). Four clusters were separated on the principal coordinates analysis (PCoA) plot, in which GM of sorafenib group were far from those of sorafenib + JHD group and JHD group (Fig. 3E). At genes level, the changes of specific microbiota were observed, and we noticed that proinflammatory GM, such as Helicobacter, saccharimonas, faecalibacterium and enterorhabdus were increased in the mice treated with sorafenib.

\subsection{JHD modulated GM composition and decreased proinflammatory microbiota.}


Following that, we paid our attention on the difference in GM between the sorafenib group and the sorafenib + JHD group. The contribution to the average dissimilarity was investigated by SIMPER procedure. Helicobacter species $(21.88 \%)$ and Lactobacillus species $(14.13 \%)$ contributed most at the genus level (Fig. 4A). Although dramatic increase of Helicobacter was shown in the sorafenib group, significance difference was not observed between these two groups(Fig. 4B). The t-test analyses showed bacteria of the genera Muribaculum, Fusicatenibacter and Dorea were enriched in the sorafenib $+\mathrm{JHD}$ group(Fig. 4C). At last, Lefse analyses was used to find biomarker, and Helicobacter species was enriched in the sorafenib group, and decreased in mice treated with the combination of sorafenib and JHD(Fig. 4D). Together, these data clearly indicated that sorafenib treatment induced expanded proinflammation microbiota which was suppressed by JHD.

\subsection{JHD decreased the infiltration of inflammatory cells and inhibited the IL-6/STAT3 pathway in tumors following the modulation of GM}

Pathological changes in the composition of the GM that lead to intestinal inflammation are a common factor for HCC[19]. The GM can gain access to the liver as a result of a chronic inflammation disease associated to dysfunction of the intestinal barrier. Following the result that proinflammatory microbiota was induced by sorafenib, we examined infiltration of inflammatory cells in the main organs, liver, lung, spleen, and tumor tissue of mice by staining (H\&E). The liver displayed the most significant changes, and dense punctate inflammatory cells were seen in the liver of sorafenib group, whereas fewer changes were observed in the sorafenib + JHD group and JHD group (Fig. 5A). The "leaky" intestinal membrane allows for translocation of bacteria-derived LPS (gram-negative bacteria) and lipoteichoic acid (LTA, derived from gram-positive bacteria)[20] which initiates inflammatory signaling pathway and ultimately leads to production of the inflammatory cytokines. IL-6/STAT3 signaling pathways link inflammation to cancer and was vital to the progression of HCC. We measured expression of the key signaling pathway IL6/janus kinase 2 (JAK2)/STAT3 at mRNA and protein levels. Expression of the cytokine and pathway activator IL-6 was downregulated in the sorafenib + JHD and JHD group. Moreover, expression of the downstream molecules JAK2 and pSTAT3 was suppressed (Fig. 5B-E). Expression of the proinflammatory mediator iNOS was increased in tumor cells in sorafenib group. (Fig. 5F, G)

\section{Discussion}

Emerging evidences show TCM acts as a concernful role in anti-tumor adjuvant therapy. JHD as a TCM formula, has been utilized in clinical for years. The application of JHD for the treatment of HCC has been studied in inducing apoptosis[6] and attenuates immunosuppressive status[21]. Sorafenib is still widely used for advanced HCC therapy. In this study, we combined JHD with sorafenib to treat HCC in a mouse model. This new strategy represents hopeful progress. First, JHD enhanced the therapeutic effect of sorafenib and alleviated reduce the adverse effect of sorafenib. Second, JHD protected sorafenib-induced increasing proinflammatory microbiota and led to downregulation of inflammation signaling. To our knowledge, this study is the first to link the composition of the intestinal microbiota of HCC with response to sorafenib treatment. 
HCC is highly lethal, and effective therapeutic treatments are still needed[5]. Immunotherapy has brought an option for cancer treatment[22], which provides survival benefit while remains low cost-effective[23]. Efficient and cost-effective treatment regimens are needed. Several studies demonstrated the dramatic effect of TCM in potently synergistic and cognitive adverse effects[24, 25], present a hopeful treatment for HCC. JHD inhibited the progression of HCC in our previous study, then we explored the synergistic effect of sorafenib in HCC.

We demonstrated that JHD in combination with anti-angiogenesis immune therapy sorafenib synergistically enhanced the anti-tumor response, thereby suppressing HCC and preventing the most common AEs(diarrhea and body weight loss) of sorafenib in BNL tumor-bearing mice. The mechanism of sorafenib-caused diarrhea is unclear and the management of diarrhea include dietary changes and preemptive use of loperamide. If severe or persistent diarrhea is unresponsive to management, interruptions/reductions of the sorafenib dose should be considered[26]. Severe AEs resulted in treatment interruption or discontinuation, which almost inevitably leads to treatment

failure and tumor progression. We showed JHD inhibited the growth of tumor as well as protected diarrhea and the body weight loss of tumor-bearing mice from sorafenib-induced, even if increasing tumor burden. Yang and colleagues present that compound kushen injection improves the therapeutic outcomes of low-dose sorafenib and avoids body weight loss[24]. JHD alleviated diarrhea of the mice treated with clinical-dose sorafenib. Together, we provided the first evidence for the combination treatment of JHD and sorafenib for HCC patients. We confirmed this finding by the investigation of expression of proliferation marker and target marker of sorafenib. Of note, we observe a significant effect of sorafenib combined with JHD on tumor cell proliferation, in spite of either sorafenib or JHD alone showing a modest suppression of proliferation in this study. Ki67 or PCNA, two common indices of proliferation, are predictive of tumor chemotherapy efficacy in terms of recurrence free survival, but Ki67 didn't show predictive of sorafenib efficacy in the phase 3 STORM trial[27]. In a previous study, JHD decreased the expression of PCNA in a H22 tumor-bearing mouse model[21]. VEGF and CD31 have been used to assess the status of vasculature and as predicters of the prognosis of cancer patients [28-30], although limited specificity. We found sorafenib further decreased the expression of VEGFA and CD31 when combined with JHD, showing a synergistical treatment of JHD.

GM plays an important role in health, disease, and responses to medication. Microbes exert indirect effects on the progression of tumor cells at distant sites or treatment responses by altering the types of circulating metabolites and immune responses, which, in turn, affect the general physiology of the host[31-33]. Nevertheless, little attention is paid to interaction between sorafenib and GM. Yamamoto K and colleagues investigated the GM of HCC patients treated with sorafenib, patients who did not suffer diarrhea had a higher abundance of Butyricimonas species and a lower abundance of Citrobacter, Peptostreptococcus, and Staphylococcaceae than that in patients with diarrhea[34]. As far as we know, we compared the composing of $\mathrm{GM}$ of mice treated with sorafenib or vehicle for the first time. We showed sorafenib induced dysbiosis and decreased anti-inflammatory GM(Muribaculum, Fusicatenibacter and Dorea) which against the efficacy of sorafenib. In previous study, low level of 
bacterias of the genera Muribaculum[35],Fusicatenibacter[36-38]and Dorea[39, 40] are associated with diarrhea and inflammation, though a part of evidence coming from non-neoplastic model. Given the critical role of chronic inflammation in progression of tumor, we speculated that sorafenib-induced changes of GM impaired the efficacy of sorafenib, which could be reversed by JHD.

In cancers, GM is mainly linked to colorectal cancer and HCC[41, 42][43]. Carcinogenesis included by bacterial pathogens in the gut mainly includes secretion of virulence factors (e.g., H.pylori), and induction of chronic inflammation; increased reactive oxygen species mediated genotoxicity(e.g., Fusobacterium nucleatum) [44]. We investigated whether the synergistic effect of JHD was due to alleviation of chronic inflammation following changes in the intestinal microenvironment. We noticed expanded lymphocyte infiltration in the liver, (but not in the lung or spleen) in sorafenib group, compared with that in the JHD group and sorafenib + JHD group. Dysbiosis and "gut leakiness" are the main contributors to liver inflammation and linked to each other. Dysbiosis may contribute to a more permeable intestinal barrier. A leaky gut enables bacterial metabolites to translocate and reach the liver readily[45]. These finding suggested JHD alleviated the inflammatory reaction of the body of mice by remodulation of GM.

As we all known, inflammatory statue is double-edged for tumor progression with activation of different pathway. We focus on the STAT3 pathway which is the central connections among inflammation, GM and HCC. Phosphorylated (p) STAT3 has been detected in 60\% of HCC patients, and STAT3-positive tumors have been deemed to be aggressive[46], and STAT3 is a major kinase-independent target of sorafenib in HCC[47]. A disturbed GM may activate IL-6/STAT3 signaling[48]. In this study, sorafenib upregulated expression of IL-6, JAK2, and pSTAT3/STAT3 and iNOS, while JHD or JHD combined with sorafenib inhibited these signaling. This finding is lined with the previous study DNMT3b/OCT4 expression confers resistance to sorafenib and a poor prognosis of HCC through regulation of IL6/STAT3 signaling[49]. Moreover, in colitis, chronic stress promotes diseases by disturbing the GM and activating IL-6/STAT3 signaling[48]. The metabolite-sensing receptor Ffar2, is a short-chain fatty acidssensing $\mathrm{G}$ protein-couple receptor that exerts immunomodulatory effects and functions in gut homeostasis and regulation of inflammation. Ffar2 activated the STAT3 axis and increases IL-22 expression[50]. Dietary supplementation with foxtail millet can ameliorate colitis-associated colorectal cancer via activation of gut receptors and suppression of the STAT3 pathway[51]. Taken together, these data suggested that JHD sensitized the therapeutic effect of sorafenib by inhibiting IL-6/STAT3 signaling following changes in GM.

Notably, there are several shortcomings in this study. First, we didn't investigate the metabolite and toxic molecule of GM which dominantly inducted chronic inflammation in mice. Second, fecal microbiota transplant, an important approach to prevent and treat disease [52], was not employed to confirmed the effect of JHD due to the limitation of this method. Oral fecal transplantation has been applied in patients and animal model, whereas inefficient survival in complex gastrointestinal environments of the microbiota due to strongly acidic gastric fluid, digestive enzymes, and bile salts[53, 54]. In summary, these evidences are still limited, and further research is needed to address these issues in greater depth. 


\section{Conclusions}

We investigated the anti-tumor effects of sorafenib and JHD in tumor-bearing mice. JHD improved the efficacy of sorafenib and significantly inhibited the growth of tumor in vivo, as well as protected tumorbearing mice from diarrhea and body weight loss induced by sorafenib. Interestingly, our study suggested JHD reshaped GM and reversed the proinflammation gut flora. The effect of JHD may be attributed to downregulated expression of the IL-6/JAK2/STAT3 pathway following changes in GM. Our investigation provided a promising approach for HCC treatment.

\section{Abbreviations}

AE adverse effects

F/B Firmicutes/Bacteroidetes

GM Gut microbiota

HCC Hepatocellular carcinoma

H\&E Hematoxylin-eosin staining

HPLC-MS High-performance liquid chromatography coupled with mass spectrometry

IHC Immunohistochemistry

IF Immunofluorescence

JAK2 Janus kinase 2

JHD Jianpi Huayu Decoction

LEfSe Linear discriminant analysis effect size

OTUs Operational taxonomic units

PcoA Principal coordinate analysis

PCNA Proliferating cell nuclear antigen

SIMPER Similarity percentages breakdown

STAT3 Signal transducer and activator of transcription-3

TCM Traditional Chinese medicine

VEGF Vascular endothelial growth 


\section{Declarations}

\section{Consent for publication}

All authors carefully reviewed the manuscript. The consent for publication was obtained from all authors.

\section{Availability of data and material}

Not applicable.

\section{Competing interests}

The authors declare that the research was conducted in the absence of any commercial or financial relationships that could be construed as a potential conflict of interest.

\section{Ethics approval and consent to participate}

The animal study was reviewed and approved by the Animal Care and Use Committee of Southern Medical University (Guangzhou, China).

\section{Author Contributions}

$\mathrm{CW}, \mathrm{XH}$ and $\mathrm{XD}$ conceived the study protocol. $\mathrm{YX}$ and $\mathrm{XD}$ wrote the manuscript. $\mathrm{XD}$ and $\mathrm{YL}$ conducted animal experiments. $X L$ and $Y L$ prepared and identified the components of JHD. $X G$ and $Y Z$ analysed and interpreted the data. CW, $\mathrm{XH}, \mathrm{JL}, \mathrm{WT}$ provided technical guidance and coordinated arrangement of funding for the whole work. All authors read and approved the final manuscript.

\section{Acknowledgements}

We thank Chao Xiang for his technical contributions in the analysis of $16 \mathrm{~S}$ data.

\section{Funding}

This study was supported by the following foundation projects: The National Natural Science Foundation of China (grant N0.81774261), and the Natural Science Foundation of Guangdong, China (grant NO. 2018A0303130315).

\section{References}


1. Bray F, Ferlay J, Soerjomataram I, Siegel RL, Torre LA, Jemal A. Global cancer statistics 2018 : GLOBOCAN estimates of incidence and mortality worldwide for 36 cancers in 185 countries. CA Cancer J Clin. 2018;68(6):394-424. 'doi:'10.3322/caac.21492.

2. Anwanwan D, Singh SK, Singh S, Saikam V, Singh R. Challenges in liver cancer and possible treatment approaches. Biochim Biophys Acta Rev Cancer. 2020;1873(1):188314. 'doi:'10.1016/j.bbcan.2019.188314.

3. Llovet JM, Ricci S, Mazzaferro V, Hilgard P, Gane E, Blanc JF, et al. Sorafenib in advanced hepatocellular carcinoma. N Engl J Med. 2008;359(4):378-90. 'doi:'10.1056/NEJMoa0708857.

4. Lu C, Rong D, Zhang B, Zheng W, Wang X, Chen Z, et al. Current perspectives on the immunosuppressive tumor microenvironment in hepatocellular carcinoma: challenges and opportunities. MOL CANCER. 2019;18(1):130. 'doi:'10.1186/s12943-019-1047-6.

5. Villanueva A. Hepatocellular Carcinoma. N Engl J Med. 2019;380(15):1450-62. 'doi:'10.1056/NEJMra1713263.

6. Huang Y, Zhou C, Wen H, Chen Y, Xie Y, Lan X, et al. Jianpi-Huayu Formula Inhibits Development of Hepatocellular Carcinoma by Regulating Expression of miR-602, Which Targets the RASSF1A Gene. INTEGR CANCER THER. 2020;19:1871119236. 'doi:'10.1177/1534735419900804.

7. Xie Y, Zhang Y, Wei X, Zhou C, Huang Y, Zhu X, et al. Jianpi Huayu Decoction Attenuates the Immunosuppressive Status of H(22) Hepatocellular Carcinoma-Bearing Mice: By Targeting MyeloidDerived Suppressor Cells. FRONT PHARMACOL. 2020;11:16. 'doi:'10.3389/fphar.2020.00016.

8. Zhu YJ, Zheng B, Wang HY, Chen L. New knowledge of the mechanisms of sorafenib resistance in liver cancer. ACTA PHARMACOL SIN. 2017;38(5):614 - 22. 'doi:'10.1038/aps.2017.5.

9. Bruix J, Raoul JL, Sherman M, Mazzaferro V, Bolondi L, Craxi A, et al. Efficacy and safety of sorafenib in patients with advanced hepatocellular carcinoma: subanalyses of a phase III trial. J HEPATOL. 2012;57(4):821-9. 'doi:'10.1016/j.jhep.2012.06.014.

10. Llovet JM, Ricci S, Mazzaferro V, Hilgard P, Gane E, Blanc JF, et al. Sorafenib in advanced hepatocellular carcinoma. N Engl J Med. 2008;359(4):378-90. 'doi:'10.1056/NEJMoa0708857.

11. Magoč T, Salzberg SL. FLASH: fast length adjustment of short reads to improve genome assemblies. BIOINFORMATICS. 2011;27(21):2957-63. 'doi:'10.1093/bioinformatics/btr507.

12. Caporaso JG, Kuczynski J, Stombaugh J, Bittinger K, Bushman FD, Costello EK, et al. QIIME allows analysis of high-throughput community sequencing data. NAT METHODS. 2010;7(5):335-6. 'doi:'10.1038/nmeth.f.303.

13. Wang Q, Garrity GM, Tiedje JM, Cole JR. Naive Bayesian classifier for rapid assignment of rRNA sequences into the new bacterial taxonomy. Appl Environ Microbiol. 2007;73(16):5261-7. 'doi:'10.1128/AEM.00062-07.

14. Karkkainen MJ, Petrova TV. Vascular endothelial growth factor receptors in the regulation of angiogenesis and lymphangiogenesis. ONCOGENE. 2000;19(49):5598 - 605. 'doi:'10.1038/sj.onc.1203855. 
15. Karki R, Kanneganti TD. Diverging inflammasome signals in tumorigenesis and potential targeting. NAT REV CANCER. 2019;19(4):197-214. 'doi:'10.1038/s41568-019-0123-y.

16. Laplane L, Duluc D, Bikfalvi A, Larmonier N, Pradeu T. Beyond the tumour microenvironment. INT J CANCER. 2019;145(10):2611-8. 'doi:'10.1002/ijc.32343.

17. Ley RE, Turnbaugh PJ, Klein S, Gordon JI. Microbial ecology: human gut microbes associated with obesity. NATURE. 2006;444(7122):1022-3. 'doi:'10.1038/4441022a.

18. Zhu Q, Xia P, Zhou X, Li X, Guo W, Zhu B, et al. Hepatitis B Virus Infection Alters Gut Microbiota Composition in Mice. Front Cell Infect Microbiol. 2019;9:377. 'doi:'10.3389/fcimb.2019.00377.

19. Jia W, Rajani $\mathrm{C}, \mathrm{Xu} \mathrm{H}$, Zheng X. Gut microbiota alterations are distinct for primary colorectal cancer and hepatocellular carcinoma. PROTEIN CELL. 2021;12(5):374 - 93. 'doi:'10.1007/s13238-02000748-0.

20. Dapito DH, Mencin A, Gwak GY, Pradere JP, Jang MK, Mederacke I, et al. Promotion of hepatocellular carcinoma by the intestinal microbiota and TLR4. CANCER CELL. 2012;21(4):504 - 16. 'doi:'10.1016/j.ccr.2012.02.007.

21. Xie Y, Zhang Y, Wei X, Zhou C, Huang Y, Zhu X, et al. Jianpi Huayu Decoction Attenuates the Immunosuppressive Status of H(22) Hepatocellular Carcinoma-Bearing Mice: By Targeting MyeloidDerived Suppressor Cells. FRONT PHARMACOL. 2020;11:16. 'doi:'10.3389/fphar.2020.00016.

22. Hou Y, Wu B. Atezolizumab plus bevacizumab versus sorafenib as first-line treatment for unresectable hepatocellular carcinoma: a cost-effectiveness analysis. Cancer Commun (Lond). 2020;40(12):743-5. 'doi:'10.1002/cac2.12110.

23. Wagle NS, Spencer JC. Challenges for Measuring Cost-effectiveness of Immunotherapy in Unresectable Hepatocellular Carcinoma. JAMA Netw Open. 2021;4(4):e215476. 'doi:'10.1001/jamanetworkopen.2021.5476.

24. Yang Y, Sun M, Yao W, Wang F, Li X, Wang W, et al. Compound kushen injection relieves tumorassociated macrophage-mediated immunosuppression through TNFR1 and sensitizes hepatocellular carcinoma to sorafenib. J IMMUNOTHER CANCER. 2020;8(1). 'doi:'10.1136/jitc-2019-000317.

25. Lv J, Jia Y, Li J, Kuai W, Li Y, Guo F, et al. Gegen Qinlian decoction enhances the effect of PD-1 blockade in colorectal cancer with microsatellite stability by remodelling the gut microbiota and the tumour microenvironment. CELL DEATH DIS. 2019;10(6):415. 'doi:'10.1038/s41419-019-1638-6.

26. Rimassa L, Danesi R, Pressiani T, Merle P. Management of adverse events associated with tyrosine kinase inhibitors: Improving outcomes for patients with hepatocellular carcinoma. CANCER TREAT REV. 2019;77:20 - 8. 'doi:'10.1016/j.ctrv.2019.05.004.

27. Pinyol R, Montal R, Bassaganyas L, Sia D, Takayama T, Chau GY, et al. Molecular predictors of prevention of recurrence in $\mathrm{HCC}$ with sorafenib as adjuvant treatment and prognostic factors in the phase 3 STORM trial. GUT. 2019;68(6):1065-75. 'doi:'10.1136/gutjnl-2018-316408.

28. Majchrzak K, Kaspera W, Szymaś J, Bobek-Billewicz B, Hebda A, Majchrzak H. Markers of angiogenesis (CD31, CD34, rCBV) and their prognostic value in low-grade gliomas. NEUROL NEUROCHIR POL. 2013;47(4):325 - 31. 'doi:'10.5114/ninp.2013.36757. 
29. Shin J, Wood LD, Hruban RH, Hong SM. Desmin and CD31 immunolabeling for detecting venous invasion of the pancreatobiliary tract cancers. PLOS ONE. 2020;15(11):e242571. 'doi:'10.1371/journal.pone.0242571.

30. Mohamed SY, Mohammed HL, Ibrahim HM, Mohamed EM, Salah M. Role of VEGF, CD105, and CD31 in the Prognosis of Colorectal Cancer Cases. J Gastrointest Cancer. 2019;50(1):23-34. 'doi:'10.1007/s12029-017-0014-y.

31. Becattini S, Taur Y, Pamer EG. Antibiotic-Induced Changes in the Intestinal Microbiota and Disease. TRENDS MOL MED. 2016;22(6):458 - 78. 'doi:'10.1016/j.molmed.2016.04.003.

32. Alexander JL, Wilson ID, Teare J, Marchesi JR, Nicholson JK, Kinross JM. Gut microbiota modulation of chemotherapy efficacy and toxicity. Nat Rev Gastroenterol Hepatol. 2017;14(6):356 - 65. 'doi:'10.1038/nrgastro.2017.20.

33. Zhang X, Coker OO, Chu ES, Fu K, Lau H, Wang YX, et al. Dietary cholesterol drives fatty liverassociated liver cancer by modulating gut microbiota and metabolites. GUT. 2021;70(4):761 - 74. 'doi:'10.1136/gutjnl-2019-319664.

34. Yamamoto K, Kuzuya T, Honda T, Ito T, Ishizu Y, Nakamura M, et al. Relationship Between Adverse Events and Microbiomes in Advanced Hepatocellular Carcinoma Patients Treated With Sorafenib. ANTICANCER RES. 2020;40(2):665 - 76. 'doi:'10.21873/anticanres.13996.

35. Dobranowski PA, Tang C, Sauvé JP, Menzies SC, Sly LM. Compositional changes to the ileal microbiome precede the onset of spontaneous ileitis in SHIP deficient mice. Gut Microbes. 2019;10(5):578 - 98. 'doi:'10.1080/19490976.2018.1560767.

36. Kim ES, Tarassishin L, Eisele C, Barre A, Nair N, Rendon A, et al. Longitudinal Changes in Fecal Calprotectin Levels Among Pregnant Women With and Without Inflammatory Bowel Disease and Their Babies. GASTROENTEROLOGY. 2021;160(4):1118-30. 'doi:'10.1053/j.gastro.2020.11.050.

37. Weis S, Schwiertz A, Unger MM, Becker A, Faßbender K, Ratering S, et al. Effect of Parkinson's disease and related medications on the composition of the fecal bacterial microbiota. NPJ Parkinsons Dis. 2019;5:28. 'doi:'10.1038/s41531-019-0100-x.

38. Crobach M, Ducarmon QR, Terveer EM, Harmanus C, Sanders I, Verduin KM, et al. The Bacterial Gut Microbiota of Adult Patients Infected, Colonized or Noncolonized by Clostridioides difficile. Microorganisms. 2020;8(5). 'doi:'10.3390/microorganisms8050677.

39. Bailey MT, Dowd SE, Galley JD, Hufnagle AR, Allen RG, Lyte M. Exposure to a social stressor alters the structure of the intestinal microbiota: implications for stressor-induced immunomodulation. BRAIN BEHAV IMMUN. 2011;25(3):397-407. 'doi:'10.1016/j.bbi.2010.10.023.

40. Rajilić-Stojanović M, Biagi E, Heilig HG, Kajander K, Kekkonen RA, Tims S, et al. Global and deep molecular analysis of microbiota signatures in fecal samples from patients with irritable bowel syndrome. GASTROENTEROLOGY. 2011;141(5):1792 - 801. 'doi:'10.1053/j.gastro.2011.07.043.

41. Sung J, Coker OO, Chu E, Szeto CH, Luk S, Lau H, et al. Gastric microbes associated with gastric inflammation, atrophy and intestinal metaplasia 1 year after Helicobacter pylori eradication. GUT. 2020;69(9):1572-80. 'doi:'10.1136/gutjnl-2019-319826. 
42. Pellicano R, Ménard A, Rizzetto M, Mégraud F. Helicobacter species and liver diseases: association or causation? LANCET INFECT DIS. 2008;8(4):254 - 60. 'doi:'10.1016/S1473-3099(08)70066-5.

43. lida N, Dzutsev A, Stewart CA, Smith L, Bouladoux N, Weingarten RA, et al. Commensal bacteria control cancer response to therapy by modulating the tumor microenvironment. SCIENCE. 2013;342(6161):967 - 70. 'doi:'10.1126/science.1240527.

44. Jia W, Xie G, Jia W. Bile acid-microbiota crosstalk in gastrointestinal inflammation and carcinogenesis. Nat Rev Gastroenterol Hepatol. 2018;15(2):111 - 28. 'doi:'10.1038/nrgastro.2017.119.

45. Schwabe RF, Greten TF. Gut microbiome in HCC - Mechanisms, diagnosis and therapy. J HEPATOL. 2020;72(2):230-8. 'doi:'10.1016/j.jhep.2019.08.016.

46. He G, Yu GY, Temkin V, Ogata H, Kuntzen C, Sakurai T, et al. Hepatocyte IKKbeta/NF-kappaB inhibits tumor promotion and progression by preventing oxidative stress-driven STAT3 activation. CANCER CELL. 2010;17(3):286 - 97. 'doi:'10.1016/j.ccr.2009.12.048.

47. Tai WT, Cheng AL, Shiau CW, Huang HP, Huang JW, Chen PJ, et al. Signal transducer and activator of transcription 3 is a major kinase-independent target of sorafenib in hepatocellular carcinoma. J HEPATOL. 2011;55(5):1041-8. 'doi:'10.1016/j.jhep.2011.01.047.

48. Gao X, Cao Q, Cheng Y, Zhao D, Wang Z, Yang H, et al. Chronic stress promotes colitis by disturbing the gut microbiota and triggering immune system response. Proc Natl Acad Sci U S A. 2018;115(13):E2960-9. 'doi:'10.1073/pnas.1720696115.

49. Lai SC, Su YT, Chi CC, Kuo YC, Lee KF, Wu YC, et al. DNMT3b/OCT4 expression confers sorafenib resistance and poor prognosis of hepatocellular carcinoma through IL-6/STAT3 regulation. J Exp Clin Cancer Res. 2019;38(1):474. 'doi:'10.1186/s13046-019-1442-2.

50. Chun E, Lavoie S, Fonseca-Pereira D, Bae S, Michaud M, Hoveyda HR, et al. Metabolite-Sensing Receptor Ffar2 Regulates Colonic Group 3 Innate Lymphoid Cells and Gut Immunity. IMMUNITY. 2019;51(5):871 - 84. 'doi:'10.1016/j.immuni.2019.09.014.

51. Zhang B, Xu Y, Liu S, Lv H, Hu Y, Wang Y, et al. Dietary Supplementation of Foxtail Millet Ameliorates Colitis-Associated Colorectal Cancer in Mice via Activation of Gut Receptors and Suppression of the STAT3 Pathway. NUTRIENTS. 2020;12(8). 'doi:'10.3390/nu12082367.

52. Smits LP, Bouter KE, de Vos WM, Borody TJ, Nieuwdorp M. Therapeutic potential of fecal microbiota transplantation. GASTROENTEROLOGY. 2013;145(5):946 - 53. 'doi:'10.1053/j.gastro.2013.08.058.

53. Costello SP, Hughes PA, Waters O, Bryant RV, Vincent AD, Blatchford P, et al. Effect of Fecal Microbiota Transplantation on 8-Week Remission in Patients With Ulcerative Colitis: A Randomized Clinical Trial. JAMA. 2019;321(2):156 - 64. 'doi:'10.1001/jama.2018.20046.

54. Cao Z, Wang X, Pang Y, Cheng S, Liu J. Biointerfacial self-assembly generates lipid membrane coated bacteria for enhanced oral delivery and treatment. NAT COMMUN. 2019;10(1):5783. 'doi:'10.1038/s41467-019-13727-9.

\section{Figures}


A

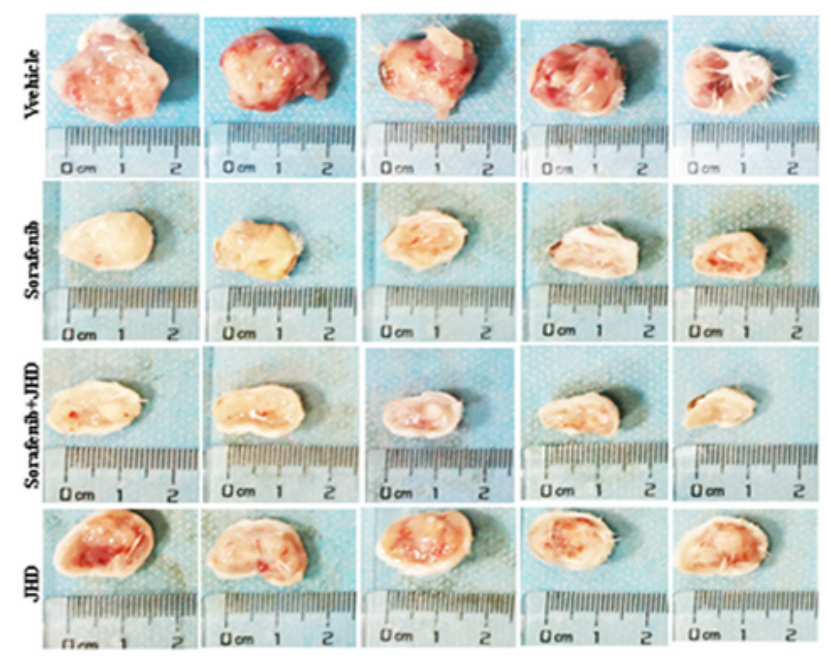

B
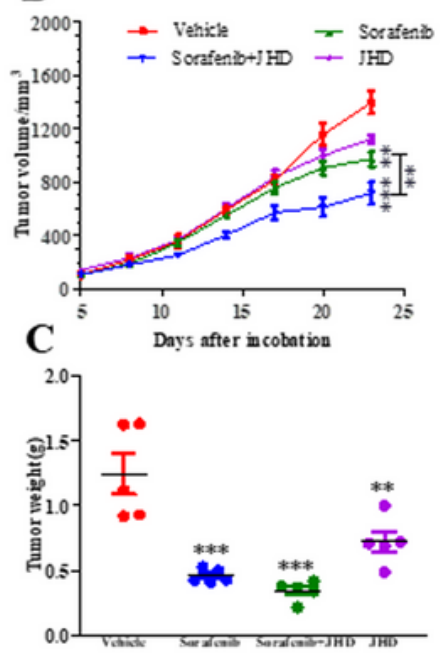

D
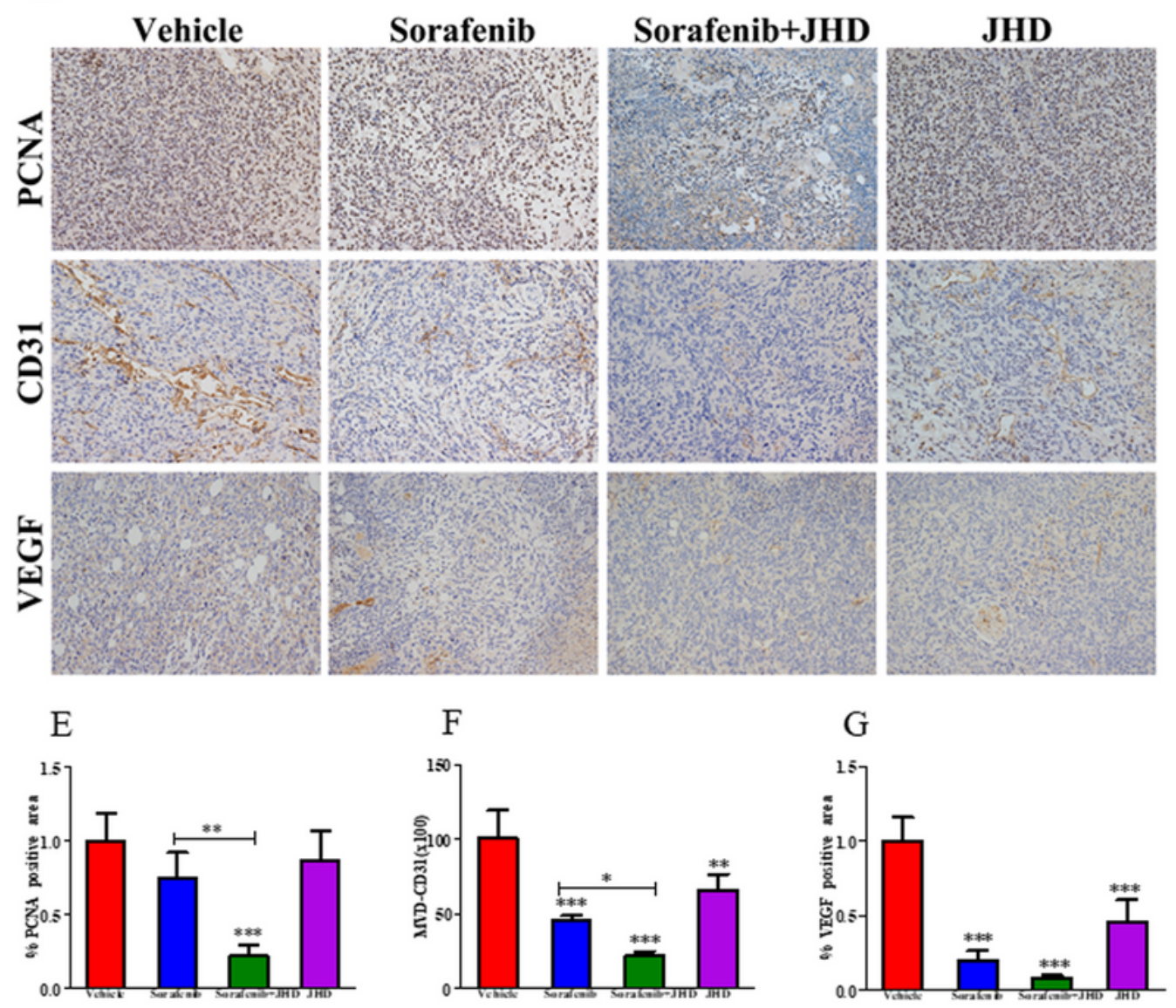

\section{Figure 1}

JHD inhibited the growth of tumor and enhanced the therapeutic effect of sorafenib in vivo. (A) Image of subcutaneous tumors. (B) Tumor volume was measured using a Vernier caliper, $\mathrm{V}=\mathrm{a} \times \mathrm{b} 2 / 2$, where $\mathrm{a}$ is the long diameter and $b$ is the short diameter, $(n=5$, two-way ANOVA). (C) Tumor weight at experimental end stage ( $n=5$, unpaired t-test). (D) Representative images of tumor sections stained immunohistochemically with PCNA, CD31, or VEGF for proliferating tumor cells and sprouting 
neomicrovessels. Images were acquired using a microscope (Olympus, Tokyo, Japan). (100x). (E) Quantification of proliferating tumor cells in four images by ImageJ. (unpaired t-test). (F) Quantification of MVD (CD31) in four immunohistochemical images. (G) Quantification of VEGF expression in five immunohistochemical images. (ANOVA for four groups comparisons and Tukey test for multiple comparisons, $\left.{ }^{*} p<0.05,{ }^{* *} p<0.01, * \star * p<0.001\right)$.
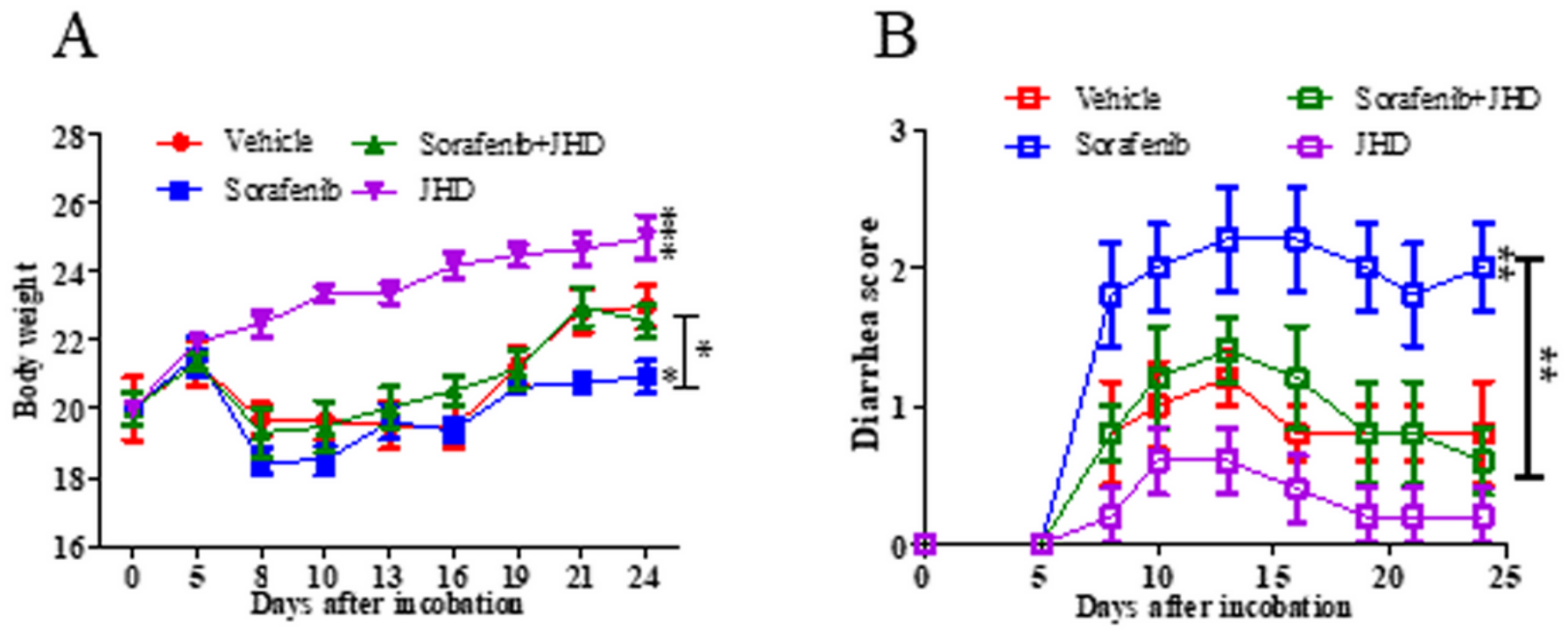

Figure 2

JHD protected occurrences of sorafenib-induced diarrhea and subsequent occurrences of body weight loss. (A) The curve of body weight. (B) Diarrhea scores(fecal: 0 marks for normal, 1 marks for wet sticky, 2 marks for visible perianal fecal, 3 marks for runny bowel) $\left(n=5\right.$; two-way ANOVA) $\left({ }^{\star} p<0.05, * \star p<0.01\right.$, $\star \star \star p p<0.001)$ 


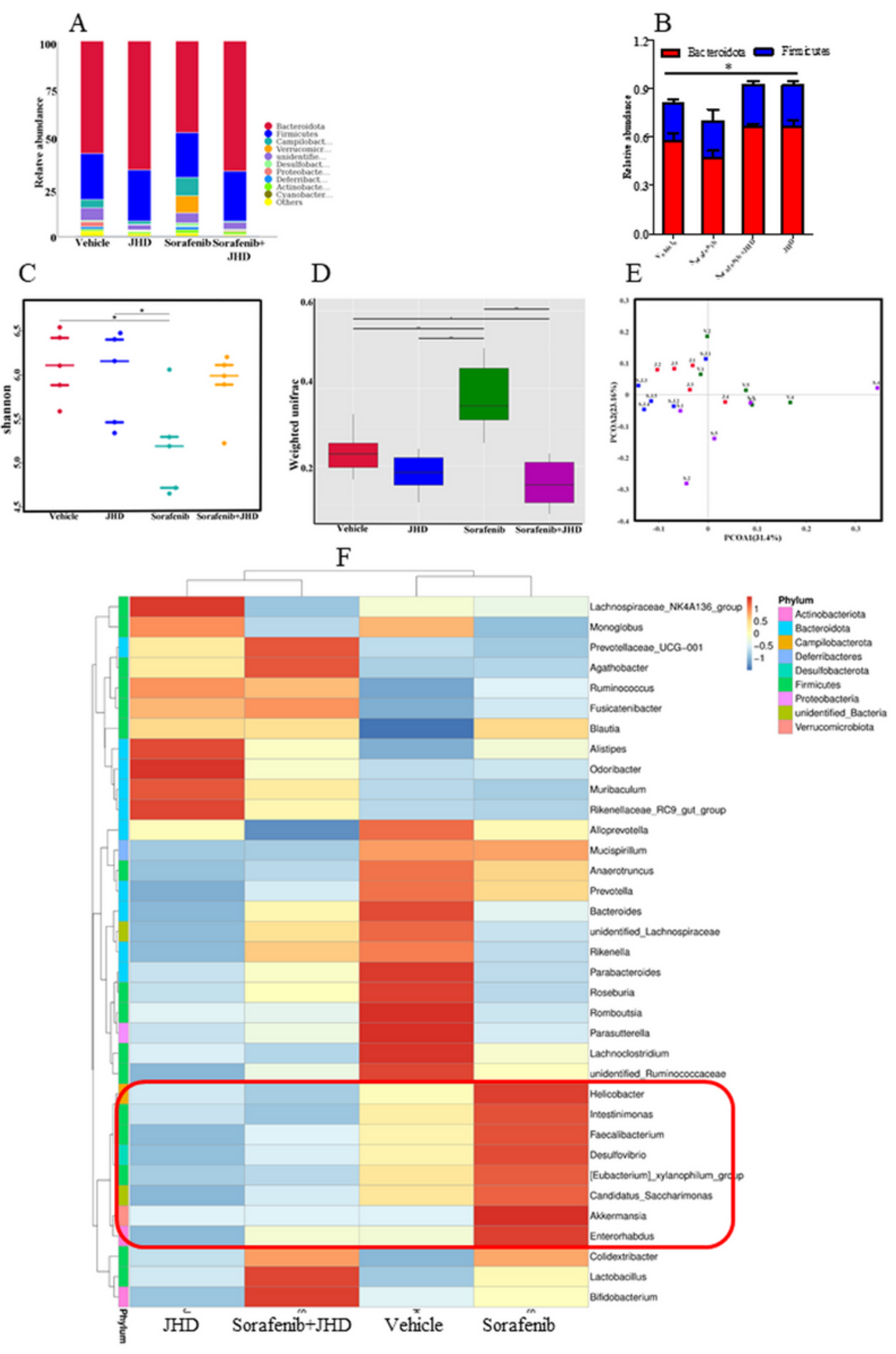

\section{Figure 3}

Sorafenib induced increased proinflammatory microbiota. (A) Relative abundance of the gut microbiota. (B) Relative abundance of Firmicutes and Bacteroidetes in the four groups. (C) Alpha analysis based on Shannon index. (D) Beta analysis based on Weighted unifrac metrics. (E) Multiple-sample principal coordinates analysis (PCoA) based on Unweighted unifrac metrics for all samples at the OTU level. (F) 
The heatmap of GM at genus level. $\left(n=5,{ }^{\star} p<0.05,{ }^{\star \star} p<0.01\right.$ ANOVA for four groups comparisons and Tukey test for multiple comparisons)
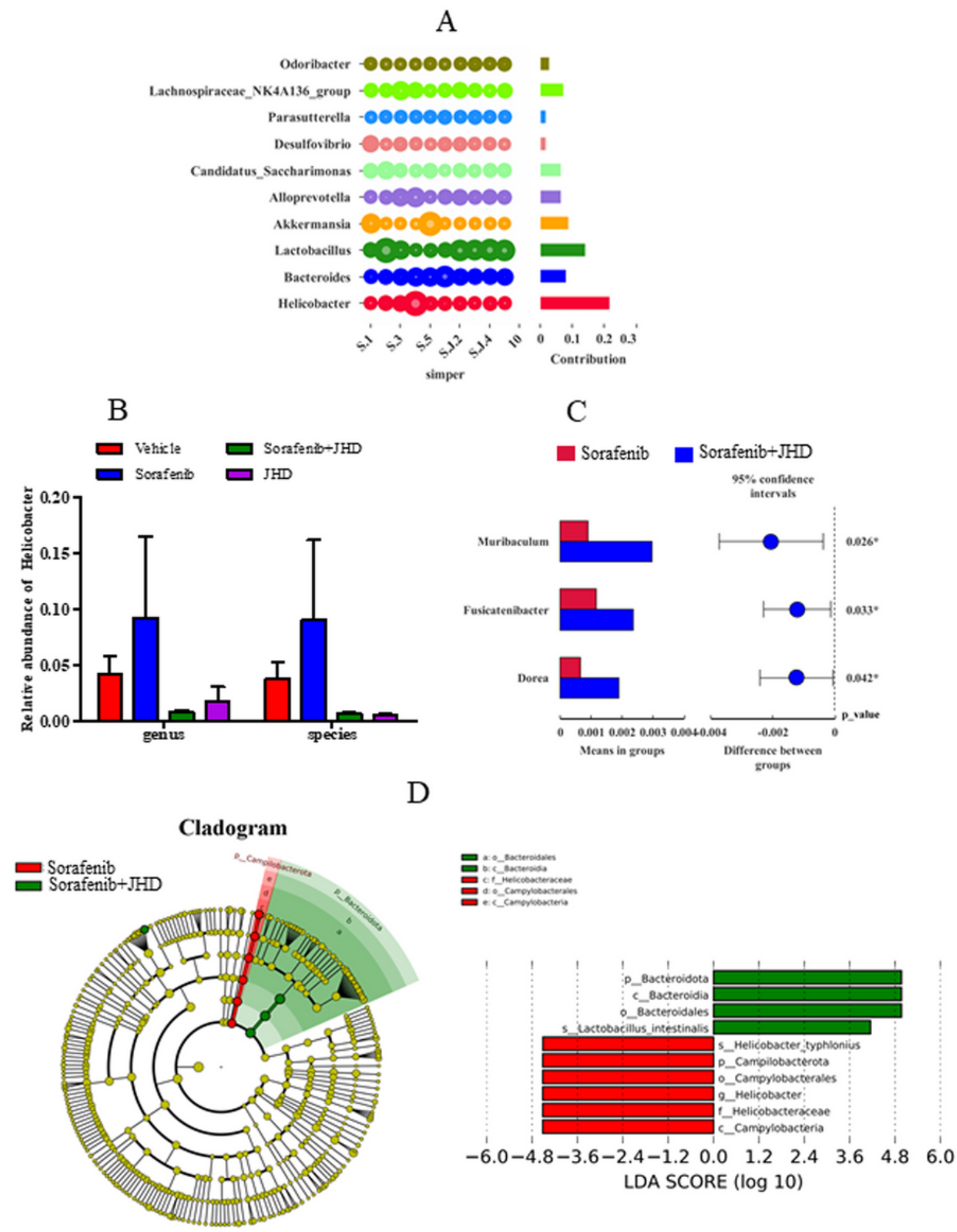

Figure 4

JHD modulated GM composition and decreased proinflammatory microbiota. (A) SIMPER analysis: quantification of the contribution of each species to the difference between the sorafenib group and sorafenib + JHD group at the genus level. (B)The relative abundance of Helicobacter at species and 
genes level. (C) t-test analysis: exploration of different species between groups at the genus level. (D)Taxonomic cladogram obtained by LEfSe. Differences are represented by the color of the most abundant class. The diameter of each circle is proportional to the taxon's abundance. Comparison of relative abundance at the phylum $(n=5$; ANOVA for four groups comparisons and Tukey test for multiple comparisons).
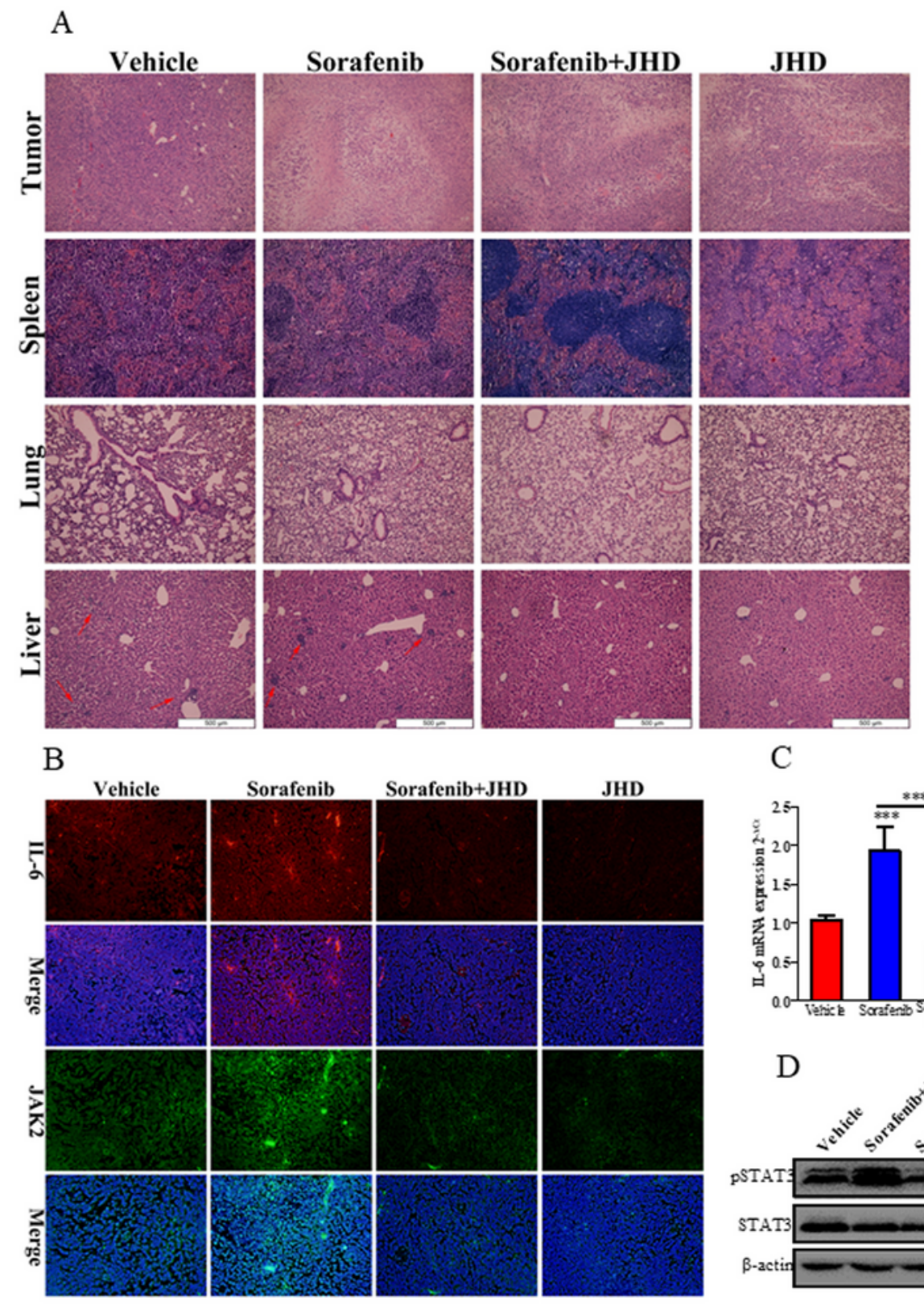

$\mathrm{E}$

$\mathrm{F}$
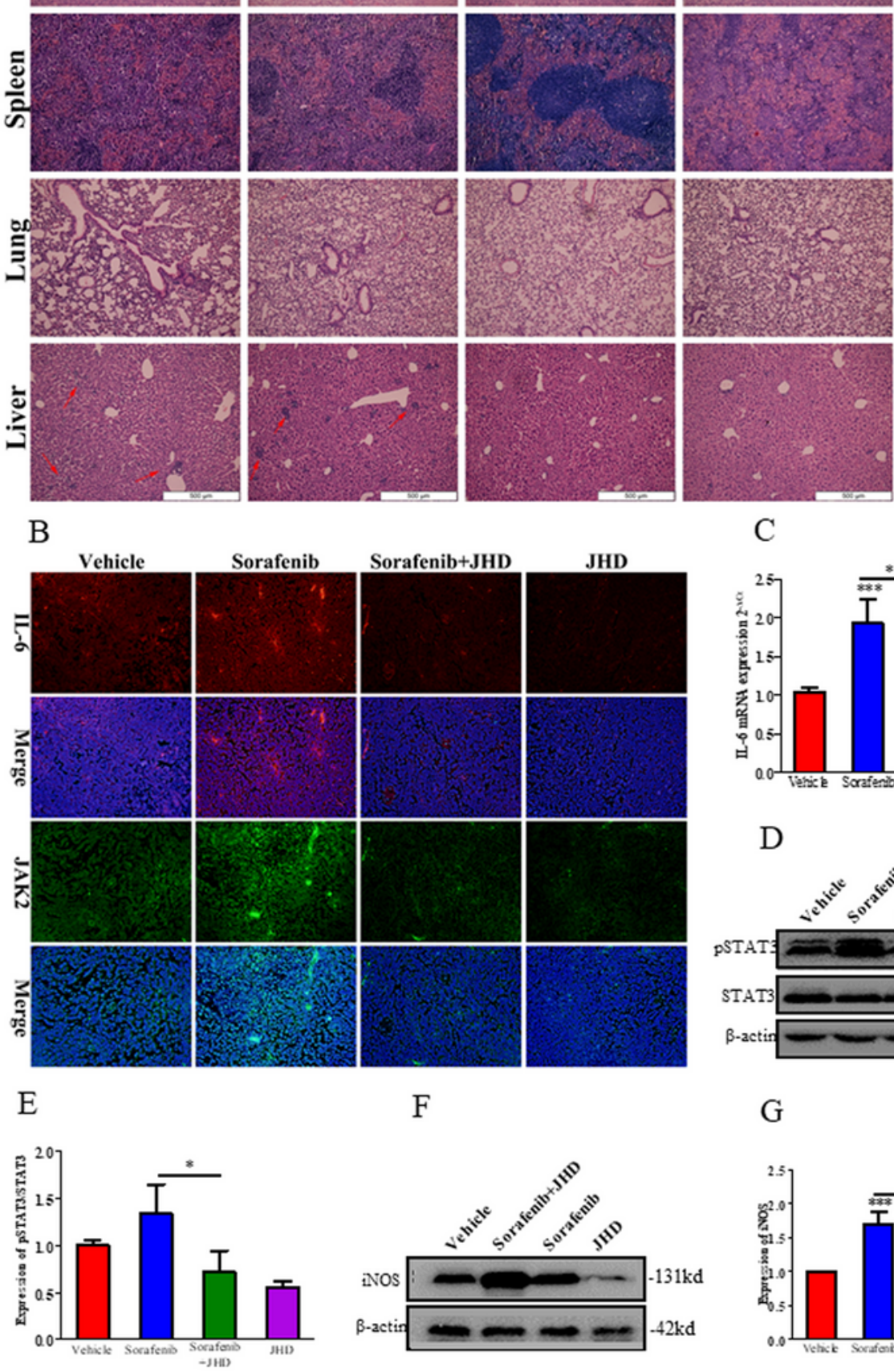

$\mathrm{C}$
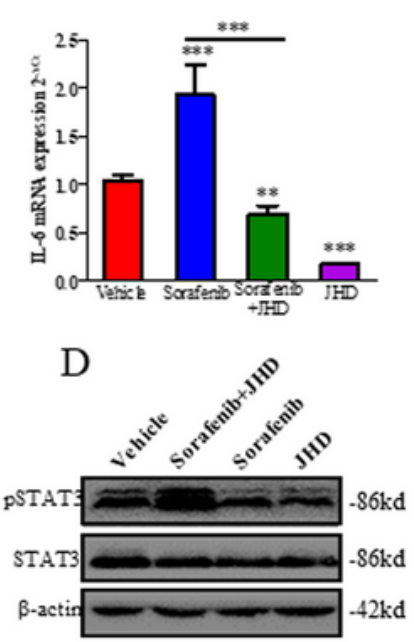

G

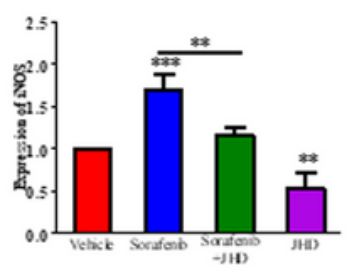

Figure 5 
JHD decreased the infiltration of inflammatory cells and inhibited the IL-6/STAT3 pathway in tumors. (A) Representative images of H\&E staining of tumor, spleen, lung, and liver (100x). (B) Representative images of immunofluorescence staining of IL-6 and, JAK2. (C) Relative quantification of expression of IL- 6 mRNA. (D, F)Protein expression of pSTAT3, STAT3 and iNOS. (E,G) Quantification of pSTAT3/STAT3 and iNOS by gray values using ImageJ. ( $F)\left(n=4 ;{ }^{*} p<0.05\right.$, ${ }^{\star \star} p<0.01$, ${ }^{\star \star \star} p<0.001$; ANOVA for four groups comparisons and Tukey test for multiple comparisons).

\section{Supplementary Files}

This is a list of supplementary files associated with this preprint. Click to download.

- Supplement1.docx

- Supplement2.xls 